\section{Bonjour la convention prestations-prix, exit la confiance médecin-patient!}

Les coûts de la santé vont à nouveau accuser une forte augmentation. Avec une somme de Fr. 30 000.- nous pouvons soigner une hépatite $\mathrm{C}$ asymptomatique mais stigmatisante et avec Fr. 70000.- (thérapie par anticorps), nous pouvons redonner espoir à un patient atteint d'un cancer du côlon en stade terminal, lui offrir une meilleure qualité de vie et prolonger son existence de quelques mois. Avec une thérapie antiTNF-alpha, nous parvenons à contrôler efficacement les symptômes d'un morbus Crohn (pour plusieurs centaines de milliers de francs pendant toute la durée de vie du patient). Les coûts de la santé doivent être ouvertement discutés, pilotés et rationnés. Mais, s'il vous plaît, pas de manière arbitraire et individuelle dans le cabinet de consultation ou au chevet du malade. La convention prestations-prix exige pourtant cela de nous, et encore davantage: le rationnement devrait être lié à notre «salaire» et réalisé au détriment de ce dernier!

Non, le rationnement doit être clairement réglé avant que le patient vienne en consultation. Il n'est pas admissible que la société laisse au médecin traitant le soin d'imposer au malade les valeurs des personnes en bonne santé. Si les vacances et l'automobile sont, pour les bien-portants, plus importantes qu'une éventuelle maladie, ce n'est pas au médecin de le faire comprendre au malade sur mandat de la société. Si ces rationnements ad hoc sont de surcroît associés à notre revenu, la confiance du patient envers son médecin sera détruite. En notre qualité de médecins, nous devons opposer un refus catégorique à cette forme de rationnement contraire à notre éthique et exiger que l'on recoure à d'autres instruments comme le catalogue des prestations, l'organisation de la médecine de pointe, etc. En raison de l'insuffisance des moyens de contrôle et compte tenu du subventionnement des hôpitaux publics par des rallonges financières, le rationnement prévu par la convention prestations-prix ne touchera que la médecine ambulatoire en cabinet privé. Le pa- tient remarquera assez vite où il peut obtenir un diagnostic et un traitement non rationnés. Les médecins indépendants seront dégradés au niveau de conseillers de la santé surqualifiés.

Je suis choqué qu'en acceptant la convention nationale prestations-prix, qui change considérablement le système, la FMH fasse du praticien indépendant un «médecin aux pieds nus» à la solde des assurances sans procéder à une discussion et une votation générale. Avant la convention prestations-prix, nous étions encore les personnes de confiance des patients qui nous consultaient et nous avions la possibilité de leur fournir une aide professionnelle.

\section{Dr A. Gut, gastro-entérologie FMH, Lucerne}

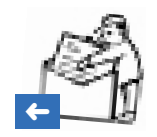

\section{Réplique}

Cher Collègue,

Je suis étonné de votre manière de comprendre les «Contrats prestations et coûts»! Bien loin d'être une menace de rationnement, il s'agit justement d'accords tarifaires qui permettent de prendre en compte les développements de la médecine (et pas seulement ceux, spectaculaires, que vous évoquez) sans qu'ils pèsent indûment sur les courbes de coûts et donc sur nos revenus un très grand progrès en fait dans nos négociations avec les assureurs. Les développements démographiques, par exemple, sont aussi pris en compte et «neutralisés».

En outre, si la FMH a signé les accords-cadres, c'est parce qu'il appartient à chaque canton ou région tarifaire de décider ensuite librement et démocratiquement de son adhésion - une politique toute de transparence!

Aucune raison donc de sombrer dans des lamentations amères, au contraire.

Le problème des CPC est bien plutôt la reculade de santésuisse qui, après les avoir signés, a presque partout abruptement changé d'option et exigé une procédure de fixation de la valeur de point par les gouvernements cantonaux; mais ça, c'est une autre histoire...

Jacques de Haller, président de la FMH 